\title{
Modelling a multi-modal logistic network with agents and dynamic graphs
}

\author{
$\underline{\text { T. Démare' }}^{\text {a }}$, S. Balev ${ }^{\text {a }}$, C. Bertelle ${ }^{\text {a }}$, A. Dutot ${ }^{\text {a }}$, D. Fournier ${ }^{\text {a }}$ and E. Sanlaville $^{\text {a }}$ \\ ${ }^{a}$ Normandie Univ, UNIHAVRE, LITIS, 76600 Le Havre, France \\ Email: thibaut.demare@univ-lehavre.fr
}

\begin{abstract}
This paper presents a model of a logistic system. Our goal is to understand how such a system (with numerous stakeholders) behaves and evolves according to different constraints or scenarios. We adopted a complex system approach which leads us to propose an agent-based model coupled with dynamic graphs. It allows us to represent the properties, constraints and behaviours at a local level of a logistic system in order to reproduce the global behaviours thanks to the simulation in a dynamic context. The simulation (which uses data about the Seine axis) allows to test different scenarios in order to understand how local decisions impact the whole system. For example, this work presents the evolution of the system at the opening of the Seine-Nord Europe Canal. Indeed, this canal is a real major project for Europe, and has numerous economical stakes. So, we first describe the traffic evolution on the multi-modal transportation network (see figures 1 to 4). Then, we observe different other measures (evolution of costs, transportation mode share). Thanks to these analyses, we show that the Seine-Nord Europe Canal should promote the use of the river barges and reduce financial costs. In the same time, it could modify the respective shares of the northern European ports.
\end{abstract}

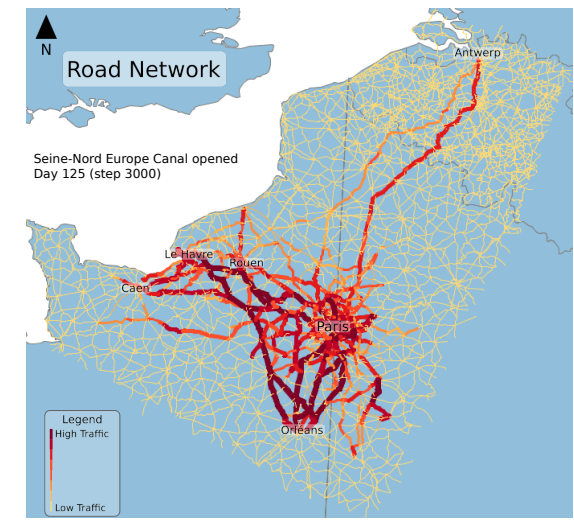

Figure 1. Road traffic (day 125) - Seine-Nord Europe Canal is opened.

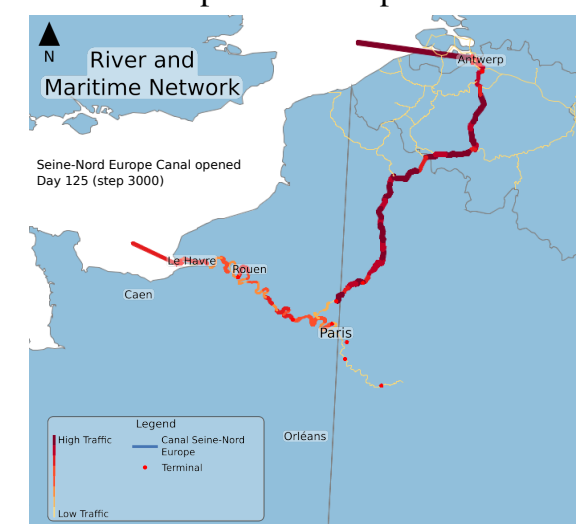

Figure 2. River traffic (day 125) - Seine-Nord Europe Canal is opened.

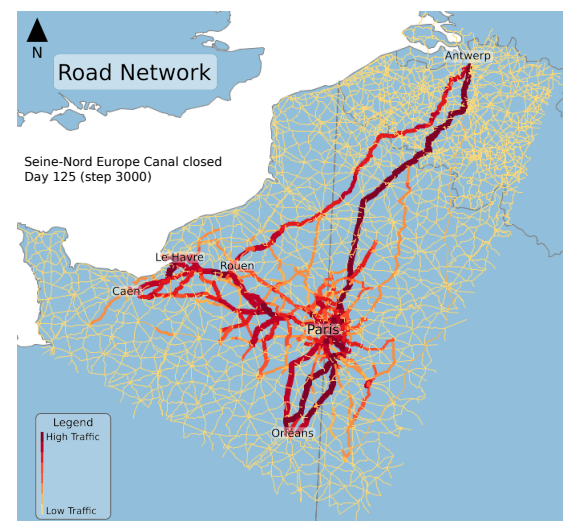

Figure 3. Road traffic (day 125) - Seine-Nord Europe Canal is closed.

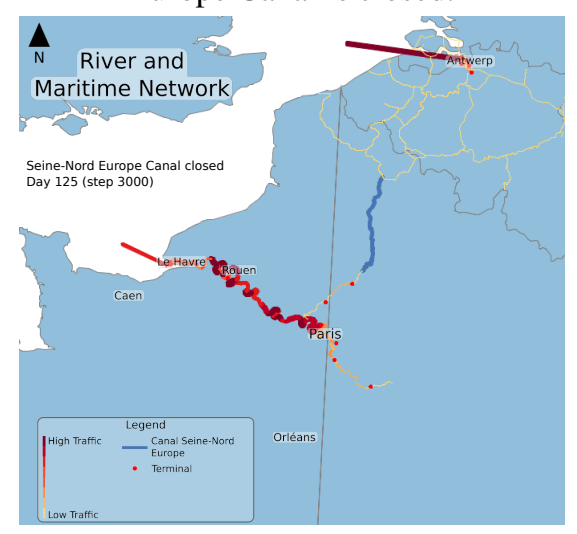

Figure 4. River traffic (day 125) - Seine-Nord Europe Canal is closed.

Keywords: Agent-based model, dynamic graph, complex system, logistic system, adaptive behaviour 


\section{INTRODUCTION AND CONTEXT}

In a logistic system, actors interact together in order to manage consistent flows of goods. They take decisions based on their own available resources (such as infrastructures, transportation vehicles,...) but they also have to deal with the constraints of the global system. The efficiency of a logistic system (including supply chains) has an important impact on its economy or on its environment. The study of such a system is therefore a major issue of logistics research. Several efforts have been made in this way, in order to optimize [Schepler et al. 2017| or to understand how flows of goods are organized [Davidsson et al., 2005].

In the literature on logistics, we can find different models, such as SMILE (Strategic Model for Integrated Logistic Evaluations) [Tavasszy et al., 1998| which uses aggregated data about flow of goods in order to extrapolate the main roads followed by the goods. We also find some models like FAME (Freight Activity Microsimulation Estimator) [Samimi et al. 2010, 2014] or TAPAS (Transportation And Production Agent-based Simulator) |Holmgren et al., 2012], which use disaggregated data. This last model is designed to simulate three actors around the Baltic sea, with a simplified transportation network. But, firstly, Tavasszy et al. |2012| highlight that these models lack of dynamism (they mostly evolve on a month or even on a year basis for each step), and secondly, the authors of FAME themselves explain that the access to the required data might be difficult. Choi et al. [2001] describe the interests to consider logistic systems as complex ones. We also showed why logistic systems should be considered as complex in Démare et al. |2017|. The complex system approach allows to model the behaviours of local entities of such a system in order to observe the evolution of the whole system thanks to auto-organization processes and emergence of properties.

The model that we present in the next section adopts an agent-based approach. We describe the actors and some infrastructures with autonomous agents of different kinds: we define how they dynamically interact, and how they behave thanks to different strategies. The transportation network is represented by dynamic graphs which can evolve in time. The model is strongly dynamic and it evolves on an hour basis with thousands of different agents. This model has been partially presented in Démare et al. [2019]. We showed that actors can adapt themselves to system modifications. We also showed how the best strategies (considering pre-defined criterion) emerge from local and distributed decisions. Nevertheless, the version of the model described in Démare et al. |2019| only used a road network to transport goods. So, our contribution in this work is the integration of a multi-modal transportation network into the simulator, and the new results this implies. Indeed, there are lots of interesting issues related to a multi-modal network. For instance, the Seine-Nord Europe Canal is a real European project which plans to link the Oise River at Compiègne (France) with the Canal Dunkerque-Escaut. After its construction (whose preliminary works have already begun), this canal will allow to connect the region of Paris with the port of Antwerp (Belgium) through waterways. The impacts of such projects are potentially important (e.g. in terms of economy or ecology) and it interests numerous local politics or actors of the logistics. So, the simulation could help to understand these impacts.

In the second section, we describe our model and some simulation results are presented in section three. We show how the system behaves when modifications of the transportation network occur and how the traffic adapts itself to these evolutions. We also present different measures made during some simulations. These results reveal that our model might be used to observe how a logistic system evolves according to some events.

\section{Modelling}

In this section, we present our model of logistic system considering import flows. First, we consider the agents directly involved in the stock management: the final consignees, the logistics service providers, the foreign goods providers, and the warehouses. More details may be found in Démare et al. [2017]; Démare et al. [2019]. In the second sub-section, we describe the agents involved in the transportation of the goods. Finally, we explain how the multi-modal network is designed.

\subsection{Agents involved in stocks management}

Figure 5 represents the actors modelled by the agents involved in stock management and how they interact together. The "final consignee" agents have local stocks. They decrease each day according to a biased random number (defined thanks to the model by Huff [1964]). A logistics service providers (LSP), chosen by the final consignee, manages the outsourced stocks. One LSP might have none or several final consignee(s), but one final consignee has only one LSP (selected randomly, biased by the distance). The goal of a LSP is to design and manage its supply network. The topology of this network is: a foreign goods provider connected to some regional warehouses, themselves connected to some local warehouses, connected to final consignee(s). 


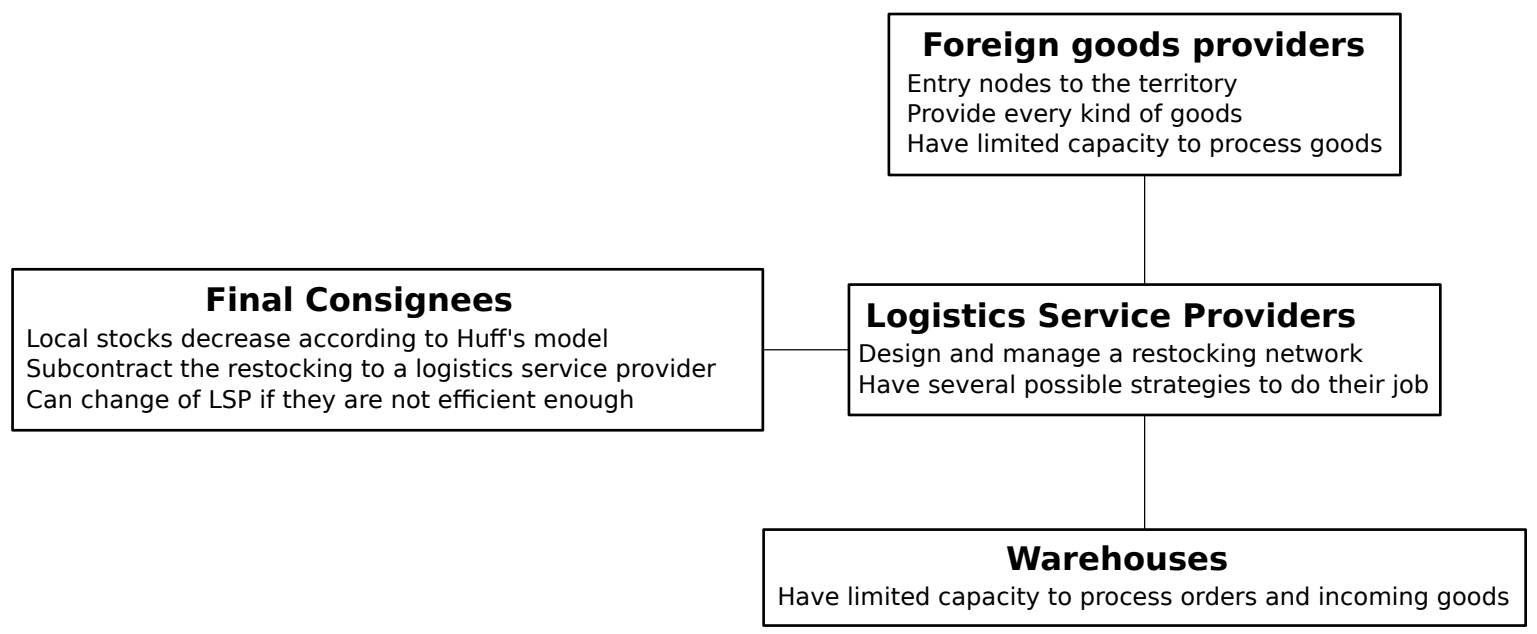

Figure 5. The actors involved in stocks management and modelled by agents.

The topology is mostly the same from a LSP to another, but the way to select a warehouse may differ. Indeed, at the initialization of the simulation, each LSP is associated to one of the four strategies of selection. The first strategy (the control strategy) simply selects randomly the warehouses. The second selects the closest warehouse to the final consignee for a local warehouse, and the one which offers the largest storage surface for the regional level. The third selects the warehouses randomly but, with a bias: according to the distance with the final consignee for the local level, and according to the storage surface for the regional level. Finally the fourth strategy makes a first filter on the warehouses, according to the distance or the surface, and then select the ones which are the most accessible in the network (in term of the accessibility index proposed by Shimbel [1953]).

Once the network is designed, each LSP monitors the stocks levels once a day. Such an agent browses each warehouse of its network. For each stock inside the warehouses of its network, the LSP determines if the current quantity of goods is too low according to a replenishment threshold. This parameter is a percentage which defines the replenishment strategy of a LSP to determine when he orders a restocking. The LSPs do not share the same replenishment threshold value. If the quantity of product is too low, then the LSP orders the replenishment to a node of higher level in the network.

During the simulation, each final consignee regularly measures the efficiency of his LSP and compares it to the average efficiency measure of every LSPs. If the LSP is not efficient enough, then the consignee can decide to choose another LSP. There are two indicators of this efficiency (inspired by the works of Teresa and Evangelos [2015]; Šrámková et al. [2015]):

- the average number of stock shortages.

- the average time used to deliver the goods to the final consignee.

- the average financial costs of deliveries.

Due to this mechanism, the final consignees will tend to keep the best LSPs and leave the worst ones. We will see in the results section that the best strategies emerge due to this behaviour.

The foreign goods providers represent the entry nodes. We consider that they aggregate all possible real foreign providers, and they can satisfy any orders of any kind of product. There are several provider agents only to model the different entry nodes of the system.

\subsection{Agents involved in goods transportation}

In our model, goods may follow a path which involves switching from one mode to another, and therefore, switching of vehicles. In this situation, this multi-modal path will have intermediate steps: the goods will have to pass through agents which model multi-modal terminals. 
Each terminal agent has the list of scheduled vehicles for the next departures. The time to load the goods on a vehicle is an attribute defined individually for each agent. This time can be different according to the type of the vehicle which will carry the goods: for instance, it is faster, to load a truck than a river barge. In the same way, there is a time to unload vehicles.

The vehicle agents can only carry a limited volume of goods simultaneously. This capacity depends on the transportation mode. The vehicles are created by the "Transporter" agents. The vehicles leave their origin at a specific date defined by the transporter agent when it creates the vehicle. A vehicle agent can only move on the network associated to its mode and to only one destination. This destination is also defined at the creation of the vehicle. Each vehicle agent has the list of goods which are scheduled to be carried, and it waits all the scheduled goods before it leaves the origin building. Therefore, vehicles may have delays. When a vehicle agent reaches its destination, the agent is destroyed (we do not manage empty vehicles, yet).

Moreover, as the vehicles move along their path (according to the speed limits), they leave, on each edge, a trace which is the amount of goods they carry. At each step, a coefficient makes decrease the trace on every edge, as the pheromones in ant colony optimization algorithms [Dorigo, 1992] which evaporate progressively. The trace is used to observe the traffic on the network: if an edge is no more used for some reasons, the evaporation process will dynamically highlight this change.

The scheduling of vehicles is managed by transporter agents. There is one transporter per transportation mode. These agents are able:

- to determinate at which date a vehicle, carrying a given volume of goods, can leave an origin node and go to a given destination node.

- to create a vehicle agent which will leave a given origin node and move to the desired destination, transporting the specified goods. The actual departure time of this vehicle is determined at this point by the transporter.

To determine the departure time of a vehicle, a transporter starts listing the vehicles already scheduled for departure on the origin node. Then, the agent applies a first filter on this list: it only keeps the vehicles whose departure time is larger than the arrival date of the goods plus the time to unload the goods from the previous vehicle and load the goods on the next vehicle. At this point, if there is a vehicle whose destination is the one of the goods, and whose remaining capacity is enough to carry the goods, then the vehicle is chosen and the departure time of the goods is the one of the vehicle. However, if there is no such vehicle, then the transporter needs to create one. To do so, the transporter computes its departure time according to the scheduled departures and to the minimal time which separate two vehicle departures from the same mode. This minimal time is of course different from one mode to another.

The last agent that we designed due to the multi-modality is the transport organizer. This agent is contacted by logistics service provider agents when they need to transport some goods. The goals of this transport organizer are to compute a multi-modal path, and then to book the necessary vehicles from the transporters. To do so, the transport organizer needs the origin and destination, and the goods to be transported. Therefore, in order to fulfil its goals, this kind of agent needs an efficient representation of a multi-modal network and some algorithms to compute these multi-modal shortest paths, described in the next section.

Figure 6 illustrates how the agents who have an active role in multi-modality interact together.

\subsection{The Multi-Modal Transportation Network}

Dynamic graphs are used to model our network. It means that its topology can be updated in real time (e.g. road works,...) [Savin, 2014; Démare et al., 2019|.

In our model, the goods can travel from and to different kinds of agents: the foreign goods providers, the terminals, the warehouses and the final consignees.

First, we have one graph for each available transportation mode. If one of the agents is connected to a transportation mode, then we create, inside of the associated graph, a node representing the agent, and we connect it to the graph by an edge. The agents "foreign goods provider" are connected to the maritime network. The agents "Warehouse" and "Final Consignee" are connected to the road network. And the agents "Terminal" are connected to at least two networks: they are all connected to the road network, but some of them are also connected to maritime network, and/or to the waterway network. 
Démare et al., Modelling a Multi-Modal Logistic Network with Agents and Dynamic Graphs

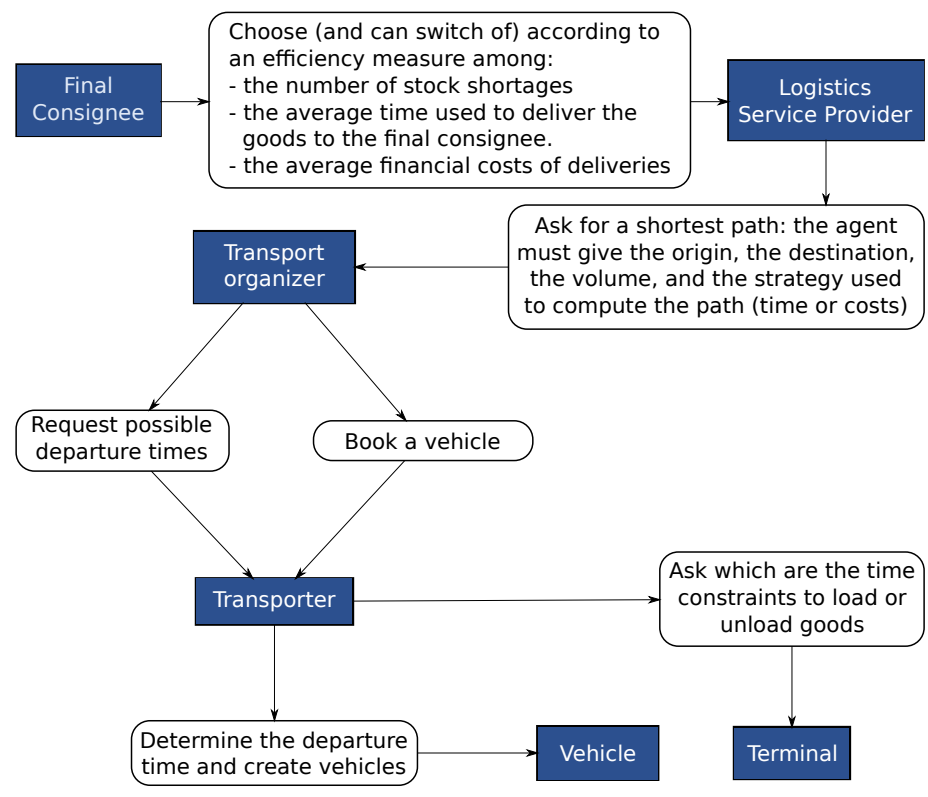

Figure 6. Interactions between the agents who have an active role in multi-modality.

Once we have a graph for each transportation mode, we have to build the multi-modal graph. This one is a multi-graph. Inside it, the only nodes are some agents of the model: the foreign goods providers, the warehouses, the final consignees, and the terminals. If two agents are connected to the same transportation mode, and if there is a path between these two agents using this mode, then we create an edge between their associated nodes. We associate a label indicating which transportation mode they represent. The multi-graph of figure 8 represents the obtained multi-modal network from networks of figure 7 .

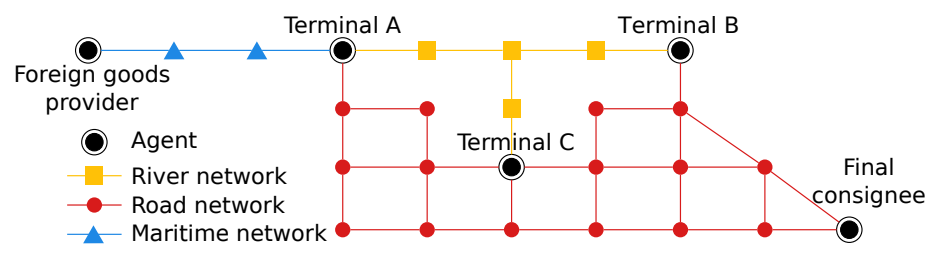

Figure 7. Example of three transportation modes connected by agents

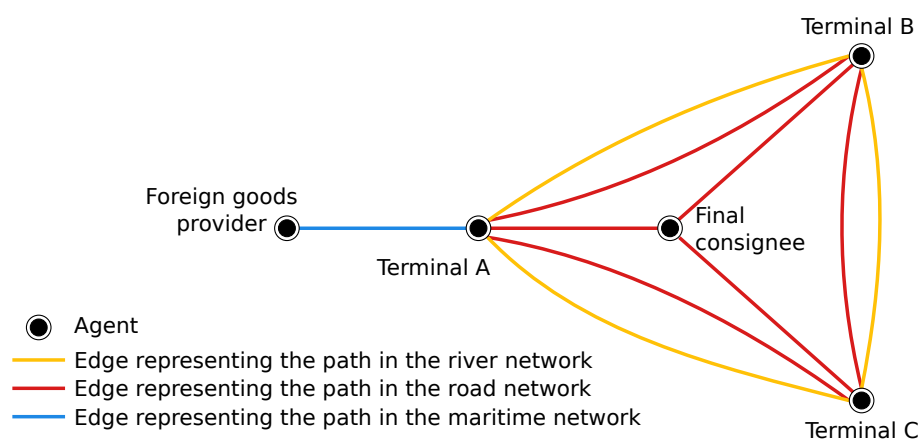

Figure 8. Model of the obtained multi-modal network based on networks from figure 7.

With this representation it is easy to compute a multi-modal shortest path. Indeed, the length of an edge (in 
terms of time) depends on two elements:

- the shortest path length (without considering the departure time and times to load and unload) on the network associated to the mode of this edge and between the two nodes of the edge. This part is a static value as long as the network is not modified. Therefore, it can be pre-computed.

- the departure time and times to load/unload goods. This second part is dynamic and depends on the previous steps of the algorithm.

Therefore, we simply adapted the Dijkstra's algorithm in order to update the length value of the leaving edges of the nodes currently explored by the algorithm according to these two parts.

\section{Results}

The simulation ${ }^{1}$ works with real data on the Seine axis in France. It includes around 3000 warehouses agents from the SITADEL2 database ${ }^{2}, 500$ final consignees agents randomly selected at each simulation among 7700 wholesalers from Sirene database ${ }^{3}$, and around 2250 LSP agents from the official list of businesses specialized in goods transportation, managed by the French Ministry for the Ecological and Solidary Transition ${ }^{4}$. The terminal agents are located thanks to the work of the Devport Team ${ }^{5}$. All of these agents are located on the Seine axis territory. The transportation network itself is built from the road network (data come from EuroGlobalMap ${ }^{6}$ ) and the river network (data come from the ETIS Project ${ }^{7}$ ). We also implemented a foreign goods provider connected to the port of Le Havre, and another one connected to the port of Antwerp. These two providers are connected to terminals thanks to a simple maritime network. We calibrated the model according to real data when they were available (such as transportation network structures, vehicles capacities, average speeds, warehouse surfaces...). For other parameters, their values were fixed to fit the actual traffic size and the different mode shares along the Seine axis. For instance, in the real world the transport mode shares indicates a river traffic near $5 \%^{8}$. In our simulations (in real conditions) the transport mode share is $94 \%$ for the road, and $6 \%$ for the river.

To obtain the results presented here we ran 16 simulations: 8 with the Seine-Nord Europe Canal (SNC) activated, and 8 others with the SNC not activated. The other parameters of the simulations are set identically. The strategies used by LSPs to select warehouses is the third (random with a bias). The replenishment threshold is $15 \%$. We choose these two strategies because we showed in Démare et al. $|2019|$ that they are the most chosen strategies when we let agents adapt their behaviours by themselves. Finally, the strategy to evaluate the LSPs by the final consignees is based on the financial costs: final consignees will tend to select another LSP if the costs are too high compared to the average costs of all other LSPs.

The figures 1 to 4 are screenshots of two different simulations and show the traffic states whether the SNC is activated or not. We can observe that the road traffic is more important between Antwerp and the Seine axis when the SNC is not activated. The SNC captures a part of the road traffic when it is activated. Indeed, the transportation mode share (which is based on the average quantities of goods measured for road and waterway modes at each step on the whole territory) is $38 \%$ for the river against $62 \%$ for the road when the SNC is not activated; and $50.1 \%$ for the river against $49.9 \%$ for the road when the SNC is activated. Moreover, the SNC has also an effect on the average financial costs. These costs increase to $25 \%$ when the SNC is not activated.

We also notice that the competition between the two ports is tighter with the SNC. Indeed, without the SNC, there are 2.9 times more LSPs who have chosen Le Havre and who are selected by some final consignees than the LSPs who have chosen Antwerp and are selected by some final consignees. But with the SNC, this value is only of 1.25. According to the parameters of our simulation and the design of our model, it is normal that Le Havre is preferred compared to Antwerp. But our simulation shows evidences that the SNC project will have influences on the European ports at an economical level if it allows to Antwerp to take shares on Le Havre. It will also have environmental effects since it should reduce the number of trucks driving to Paris area.

\footnotetext{
${ }^{1}$ The source code of the implementation of this model may be found at: https://github.com/ThibautDemare/DALSim

${ }^{2} \mathrm{http}: / / \mathrm{www}$. statistiques.developpement-durable.gouv.fr/sources-methodes/enquete-nomenclature/1542/0/base-sitdel2.html

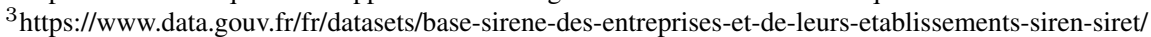

${ }^{4}$ https://www.ecologique-solidaire.gouv.fr/liste-des-entreprises-inscrites-au-registre-electronique-national-des-entreprises-transportroute-et

${ }^{5}$ It is a multidisciplinary research team who works on the study of the Seine axis' logistic system. Website: http://www.projetdevport.fr/en/

' $\mathrm{http}: / /$ professionnels.ign.fr/egm

${ }^{7}$ https://www.tmleuven.be/en/project/etisplus

${ }^{8}$ Source: http://www.cci-paris-idf.fr/sites/default/files/etudes/pdf/documents/prise_de_position_ccir_axe_seine_20170726_v2.pdf
} 


\section{Conclusion}

To conclude, we described in this article an agent based model coupled with dynamic graphs to represent and simulate a logistic system. We adopted a complex system approach in order to model the local behaviours and properties of logistic systems. This model has a multi-modal transportation network. It allows us to observe its evolution according to different scenarios. As an example, we observed how the system evolves when we activate, or not, the Seine-Nord Europe Canal. We showed that the economical and environmental stakes of such a project are significant. However, the results from the simulations have limits and do not totally reflect the reality. Indeed, the simulations are affected by the efficiency measure used by final consignees which is based on financial costs, but the model does not consider other factors such as reputation, habits,... But it might be a real challenge to include such factors since they are often based on subjective criteria.

As a perspective, we would like to check the model validity comparing our simulation outputs with the aggregated data from the ETIS project. Next, we will explore a bit further the capacities of our model with its multi-modal transportation network. Indeed, we want to study the effects and the viability of the short sea shipping between the local maritime ports of the Seine axis thanks to an extended maritime network.

\section{ACKNOWLEDGements}

This work is part of the project "CLASSE2", co-financed by the European Union thanks to the European grant of regional development and by the Normandie region.

\section{REFERENCES}

Choi, T. Y., K. J. Dooley, and M. Rungtusanatham (2001). Supply networks and complex adaptive systems: control versus emergence. Journal of Operations Management 19(3), 351 - 366.

Davidsson, P., L. Henesey, L. Ramstedt, J. Törnquist, and F. Wernstedt (2005). An analysis of agent-based approaches to transport logistics. Transportation Research Part C: Emerging Technologies 13(4), 255 271.

Démare, T., C. Bertelle, A. Dutot, and D. Fournier (2019, March). Adaptive behavior modeling in logistic systems with agents and dynamic graphs. ACM Trans. Auton. Adapt. Syst. 13(3), 15:1-15:25.

Démare, T., C. Bertelle, A. Dutot, and L. Lévêque (2017). Modeling logistic systems with an agent-based model and dynamic graphs. Journal of Transport Geography 62(Supplement C), 51 - 65.

Dorigo, M. (1992). Optimization, Learning and Natural Algorithms. Ph. D. thesis, Politecnico di Milano, Italy.

Holmgren, J., P. Davidsson, J. A. Persson, and L. Ramstedt (2012). Tapas: A multi-agent-based model for simulation of transport chains. Simulation Modelling Practice and Theory 23, $1-18$.

Huff, D. L. (1964). Defining and estimating a trading area. Journal of Marketing 28(3), pp. 34-38.

Šrámková, E., E. Niko, P. Kolář, and J. Huňak (2015, May). Decision-making factors leading to customers' satisfaction in container transportation. In 4th International Conference on Advanced Logistics and Transport (ICALT), pp. 105-110.

Samimi, A., A. Mohammadian, and K. Kawamura (2010). A behavioral freight movement microsimulation model: method and data. Transportation Letters 2(1), 53-62.

Samimi, A., A. Mohammadian, K. Kawamura, and Z. Pourabdollahi (2014). An activity-based freight mode choice microsimulation model. Transportation Letters 6(3), 142-151.

Savin, G. (2014, January). Intelligence en essaim pour la distribution de simulations dans un écosystème computationnel. $\mathrm{Ph}$. D. thesis, Université du Havre.

Schepler, X., S. Balev, S. Michel, and E. Sanlaville (2017). Global planning in a multi-terminal and multimodal maritime container port. Transportation Research Part E: Logistics and Transportation Review 100, $38-62$.

Shimbel, A. (1953). Structural parameters of communication networks. The bulletin of mathematical biophysics 15(4), 501-507.

Tavasszy, L. A., K. Ruijgrok, and I. Davydenko (2012). Incorporating logistics in freight transport demand models: state-of-the-art and research opportunities. Transport Reviews 32(2), 203-219.

Tavasszy, L. A., B. Smeenk, and C. J. Ruijgrok (1998). A dss for modelling logistic chains in freight transport policy analysis. International Transactions in Operational Research 5(6), 447 - 459.

Teresa, G. and G. Evangelos (2015, May). Importance of logistics services attributes influencing customer satisfaction. In 4th International Conference on Advanced Logistics and Transport (ICALT), pp. 53-58. 